\title{
Effect of the Degree of Polymerization of Fructans on Ex Vivo Fermented Human Gut Microbiome
}

\author{
Erola Astó ${ }^{1,2}$, Iago Méndez ${ }^{1}$, Maria Rodríguez-Prado ${ }^{3}$, Jordi Cuñé ${ }^{1, \dagger}$, Jordi Espadaler ${ }^{1, *}$ and \\ Andreu Farran-Codina ${ }^{2}$ (1) \\ 1 AB-Biotics, S.A, ESADE Creapolis, Av. Torre Blanca, 57, E-08172 Sant Cugat del Vallès (Barcelona), Spain; \\ asto@ab-biotics.com (E.A.); mendez@ab-biotics.com (I.M.); jordicune@gmail.com (J.C.) \\ 2 Department of Nutrition, Food Science, and Gastronomy, XaRTA-INSA, Faculty of Pharmacy, University of \\ Barcelona, Campus de l'Alimentació de Torribera, Av. Prat de la Riba, 171, E-08921 Santa Coloma de \\ Gramenet, Spain; afarran@ub.edu \\ 3 Animal Nutrition and Welfare Service (SNIBA), Building V. Office V0-308, Autonomous University of \\ Barcelona, C/ Travessera dels Turons s/n, E-08193 Bellaterra (Barcelona), Spain; \\ maria.rodriguez.prado@uab.cat \\ * Correspondence: espadaler@ab-biotics.com; Tel.: +34-935-800-236 \\ + Present address: Devicare, S.A, Eureka Building, UAB Research Park, Av. Can Domènech s/n, E-8193 \\ Cerdanyola del Vallès (Barcelona), Spain.
}

Received: 28 April 2019; Accepted: 4 June 2019; Published: 7 June 2019

\begin{abstract}
Prebiotic supplements are used to promote gastrointestinal health by stimulating beneficial bacteria. The aim of this study was to compare the potential prebiotic effects of fructans with increasing degrees of polymerization, namely fructooligosaccharides (FOS) and inulins with a low and high polymerization degree (LPDI and HPDI, respectively), using an ex vivo fermentation system to simulate the colonic environment. The system was inoculated with pooled feces from three healthy donors with the same baseline enterotype. Changes in microbiota composition were measured by $16 \mathrm{~S}$ metagenomic sequencing after 2, 7, and 14 days of fermentation, and acid production was measured throughout the experiment. Alpha-diversity decreased upon inoculation of the ex vivo fermentation under all treatments. Composition changed significantly across both treatments and time (ANOSIM $p<0.005$ for both factors). HPDI and LPDI seemed to be similar to each other regarding composition and acidification activity, but different from the control and FOS. FOS differed from the control in terms of composition but not acidification. HDPI restored alpha-diversity on day 14 as compared to the control (Bonferroni $p<0.05$ ). In conclusion, the prebiotic activity of fructans appears to depend on the degree of polymerization, with LPDI and especially HPDI having a greater effect than FOS.
\end{abstract}

Keywords: prebiotic; inulin-type fructan; fructooligosaccharide; polymerization degree; metagenomics; microbiome

\section{Introduction}

It is generally accepted that the bacterial community in the human gastrointestinal tract has a great impact on intestinal functionality and human health.The colon is the most colonized region within the gastrointestinal tract, where $10^{11}-10^{12}$ cells $/ \mathrm{mL}$ have been detected [1,2]. The microbes in our bodies have been estimated to encode 100-fold more unique genes than our own genome [3,4]. High-quality data from the US Human Microbiome Project (HMP) [5], European Metagenomics of the Human Intestinal Tract (MetaHIT) [6] and several other studies have now demonstrated the beneficial role of the normal gut microbiota in health down to the genetic level [6,7]. Most bacterial species colonizing the human gastrointestinal tract belong to the phyla Firmicutes and Bacteroidetes, while species of the phyla Actinobacteria, Proteobacteria, and Verrucomicrobia exist in lower numbers. The 
number of bacterial species within the human intestinal microbiota has often been estimated to be in the range of 500 to over 1000 species [8]. In a healthy state, they contribute nutrients and energy to the host via the fermentation of nondigestible dietary components in the large intestine, and a balance is maintained with the host's metabolism and immune system $[9,10]$.

The prebiotic concept was first defined in 1995 as a "non-digestible food ingredient that beneficially affects the host by selectively stimulating the growth and/or activity of one or a limited number of bacteria already resident in the colon" [11]. The International Scientific Association for Probiotics and Prebiotics (ISAPP) recently published a consensus statement to update the definition and scope of prebiotics. The statement defines a prebiotic as "a substrate that is selectively utilized by host microorganisms conferring a health benefit" [12]. The scope of this updated definition is therefore wider, as it implies that the modulatory effect of prebiotics is not necessarily limited to gastrointestinal microbiota (i.e., it can be administered to microbiota-colonized sites other than the gut). Furthermore, the ISAPP consensus statement emphasizes that the definition of prebiotics should not be limited to carbohydrate-based compounds, but should apply to any compound that can be used by the microbiota to confer a health benefit. Some examples are polyphenols and polyunsaturated fatty acids. The document also addresses the difficulties involved in providing definitive proof of causality when assessing the beneficial effects of a prebiotic. Still, it states that the health benefits of a prebiotic must be confirmed in the target host for its intended use and should always be mediated by the microbiota.

Inulin-type fructans (ITF) are the best-documented oligosaccharides for their effect on intestinal Bifidobacteria and are considered to be important prebiotic substrates [13]. Fructans can also be described by their polymerization degree (PD). Inulin-type fructans consisting of DP $\geq 10$ units are considered long chain, while inulin-type fructans with a DP of $<10$ are considered short chain [14]. Nutritional studies have recommended a combination of inulins with different chain lengths in order to maximize their fermentative and prebiotic effects [13]. Beneficial effects of inulin and/or oligofructose on the intestinal microbiota, have been demonstrated in adults, such as increasing Bifidobacteria and Lactobacilli, as well as increasing butyrate production [15-17]. Moreover, the prebiotic activity of inulin and oligofructose seems to translate into beneficial health effects such as improved glycemic control, reduced plasma triglycerides, improved barrier function, and decreased oxidative stress [14]. However, not all fructans seem to produce the same effects on the gut microbiota [18-20].

Ex vivo fermentation models are considered reliable tools to assess how microbial populations are altered by gastrointestinal environments such as prebiotics [21]. The common purpose of ex vivo gut fermentation models is to cultivate a complex intestinal microbiota under controlled environmental conditions with the aim of carrying out microbial modulation and metabolism studies. Although there are several well-studied and validated complex in vitro models of human microbiota (e.g., the Simulator of the Human Intestinal Microbial Ecosystem (SHIME) [22,23], the TNO Gastrointestinal Model (TIM-2) [24] and the Three-Stage Compound Continuous Culture System [25], we were interested in performing a simpler continuous culture system with one single stage.

Several studies have classified the human intestinal microbiota into three enterotypes in order to reduce the complexity and facilitate the identification of microbiota compositional patterns [26,27]: enterotype 1, which has Bacteroides as its best indicator; enterotype 2, which is driven by Prevotella, a genus whose abundance is inversely correlated with Bacteroides; and enterotype 3, which is distinguished by a significant presence of Firmicutes especially of the Ruminococcus genus [26-28]. However, this classification should be treated with caution, as some authors have suggested that microbiota could be divided into two enterotypes, where Bacteroides enterotype was fused with the less well distinguished Firmicutes enterotype while other authors have proposed four enterotypes, two of theme resembling previously the described Bacteroides and Prevotella enterotypes, while the other two are a more complex mixture with lower levels of Bacteroides, one dominated by a variety of populations affiliated within the Firmicutes phylum and the other combining higher levels of Faecalibacterium and Ruminococcaceae (both belonging to the Firmicutes phylum) with Alistipes (which belongs to the Bacteroidetes phylum) [28,29]. 
The objective of the study was to evaluate how the polymerization degree influences the prebiotic effect of various fructans (FOS and two inulins with differing degrees of polymerization) in an ex vivo fermented human microbiome, under conditions that simulated the transverse colon, taken from the Macfarlane model [25,30].

\section{Materials and Methods}

\subsection{Fructans Analyzed}

Three commercial fructans with differing average fructosyl chain lengths (i.e., degree of polymerization) were chosen for this study.

1. Oligofructose (FOS): Orafti ${ }^{\circledR}$ P95 (BENEO-Orafti S.A., Tienen, Belgium), a short-chain inulin obtained through partial enzymatic hydrolysis and consisting of fructose units joined by $\beta(2-1)$ linkages, with an end-standing glucose unit and an average polymerization degree of two to eight units.

2. Low-polymerization-degree inulin (LPDI): Orafti ${ }^{\circledR}$ GR (BENEO-Orafti S.A., Tienen, Belgium), a granulated powder extracted primarily from chicory and consisting of fructose units joined by $\beta(2-1)$ linkages, with an end-standing glucose unit and an average polymerization degree of $\geq 10$.

3. High-polymerization-degree inulin (HPDI): Orafti ${ }^{\circledR}$ HPX (BENEO-Orafti S.A., Tienen, Belgium), a high-performance inulin derived from chicory and consisting of fructose units joined by $\beta(2-1)$ linkages, with an end-standing glucose unit and an average polymerization degree of $\geq 23$.

The concentration of each prebiotic in each fermentation vessel was $12 \mathrm{~g} / \mathrm{L}$.

\subsection{Donor Information}

Inclusion criteria of volunteers were as follows: healthy young adults aged 18-30 years, a BMI of between 19 to $29.9 \mathrm{~kg} / \mathrm{m}^{2}$, and type-3 or type- 4 stools over the previous $72 \mathrm{~h}$, as per the Bristol stool scale [31]. Exclusion criteria were as follows: infectious diseases in the three months prior to fecal donation, antibiotic consumption in the three months prior to donation, blood in the donation, and alcohol intake $72 \mathrm{~h}$ prior to donation. Participants were asked to fill out a $72 \mathrm{~h}$ dietary recall form and a food frequency questionnaire (Supplementary Table S1). Donors were required to consume a varied diet rich in fruit and vegetables (with a minimum of three portions of vegetables and two portions of fruit per day).

All subjects gave their informed consent for inclusion before they participated in the study. The study was conducted in accordance with the Declaration of Helsinki, and the protocol was approved by the Ethics Committee of the University of Barcelona (IRB00003099).

\subsection{Fecal Sample Collection and Inoculum Preparation}

Fecal samples were collected (a minimum of $150 \mathrm{~g}$ from each donor) and kept in anaerobic conditions with Oxoid AnaeroGen (Thermo Scientific). The samples were homogenized by dissolution in glycerol/PBS $(10 \% w / v)$ directly after collection to maximize preservation for the subsequent stages. The samples were frozen at $-20^{\circ} \mathrm{C}$ and stored in ready-to-use aliquots in the ex vivo fermentation [32,33]. Prior to fermentation, one aliquot from each donor was incubated for $1 \mathrm{~h}$ at $37^{\circ} \mathrm{C}$.

\subsection{Fermentation}

Fermentation was carried out in a single-flow in vitro digestion system that simulated the transverse colon conditions defined previously $[25,30]$. The system consisted of eight independent units (500 $\mathrm{mL}$ amber glass jars), in which the fermentation conditions were monitored and controlled. The $\mathrm{pH}$ of the culture medium was maintained at $6.2 \pm 0.2$ by means of a $\mathrm{pH}$ meter (model PH 28, Crison Instruments S.A., Barcelona, Spain) connected to two peristaltic minipumps (8 RPM, 100 series pump; Williamson, Southwick, UK) to regulate the addition of $\mathrm{HCl} 2 \mathrm{~N}$ or $\mathrm{NaOH} 3 \mathrm{~N}$. Temperature 
was monitored with a probe connected to a digital temperature controller (Autonics, Busan, South Korea) and maintained at $37^{\circ} \mathrm{C} \pm 0.5$ with a circulating water bath (PolyScience, Niles, IL, USA). Anaerobic fermentation conditions were achieved by constant flushing of $\mathrm{O}_{2}-$ free $\mathrm{N}_{2}$ gas. Liquid retention time was $48 \mathrm{~h}(2.083 \% / \mathrm{h}$ dilution rate) by means of two precision peristaltic pumps, one for the culture medium input and one for the fermentation medium output (Masterflex Digital Console Drive; Cole-Parmer, Vernon Hills, IL, USA). All equipment and consumables used in the fermentation process were sterilized before the experiment and sampling started.

The artificial colon simulator was fed a complex mixture of nutrients defined by Macfarlane and widely used in the literature $[25,34,35]$. This mixture was used as our basal medium, either supplemented with the fructans or alone as a control. The potentially interesting fructans were tested after being added to the Macfarlane composition, while the basal medium alone was used as a control treatment. An aliquot of $60 \mathrm{~mL}$ of the prepared fecal inoculum was added to each fermenter, together with $340 \mathrm{~mL}$ of the basal medium. The experiments were carried out in parallel in eight anaerobic jars (two replicas for each treatment with the basal medium or the basal medium plus fructans).

\subsection{Metagenomics Analysis}

\subsubsection{DNA Extraction}

Bacterial DNA was extracted from $0.2 \mathrm{~g}$ of each sample using the PowerFecal ${ }^{\mathrm{TM}}$ DNA isolation kit (MO BIO, Carlsbad, CA, USA), in accordance with manufacturer's instructions. DNA samples $(100 \mu \mathrm{L})$ were stored at $-20{ }^{\circ} \mathrm{C}$ until further processing. To assess for contamination from the laboratory or reagents, blank samples were processed at the same time. Samples obtained from the fermenters were processed for DNA extraction upon collection.

\subsubsection{PCR Amplification and Massive Sequencing}

The V4 region of the $16 \mathrm{~S}$ rRNA gene was amplified with the widely used primer pair F515 (5' GTGYCAGCMGCCGCGGTAA-3') and R806 (5'-GGACTACNVGGGTWTCTAAT-3'). Both primers included sequencing adaptors at the $5^{\prime}$ end and forward primers were tagged with different barcodes to pool samples in the same sequencing reaction. The PCR mixture $(25 \mu \mathrm{L})$ contained $2 \mu \mathrm{L}$ of DNA template (5 ng), $5 \mu \mathrm{L}$ of $5 \times$ Phusion $^{\circledR}$ High-Fidelity Buffer, $2.5 \mu \mathrm{L}$ of dNTPs $(2 \mathrm{mM}), 0.2 \mu \mathrm{M}$ of each primer and $0.5 \mathrm{U}$ of Phusion ${ }^{\circledR}$ Hot Start II Taq Polymerase (Thermo Fisher, Waltham, MA, USA).

The PCR thermal profile consisted of an initial denaturation at $98^{\circ} \mathrm{C}$ for $30 \mathrm{~s}$, followed by 30 cycles at $98{ }^{\circ} \mathrm{C}$ for $15 \mathrm{~s}, 55{ }^{\circ} \mathrm{C}$ for $15 \mathrm{~s}, 72{ }^{\circ} \mathrm{C}$ at $20 \mathrm{~s}$ and a final step at $72{ }^{\circ} \mathrm{C}$ for $7 \mathrm{~min}$. To assess possible reagent contamination, each PCR reaction included a no template control (NTC) sample. For each amplicon, the quality and quantity were assessed with an Agilent 2100 Bioanalyzer and a Qubit ${ }^{\mathrm{TM}}$ fluorometer, respectively.

Each sequencing pool included 40 barcoded samples that were sequenced on an Ion Torrent Personal Genome Machine (PGM) with the Ion 318 Chip Kit v2 and the Ion PGM ${ }^{\mathrm{TM}}$ Sequencing 400 Kit (Life Technologies, Carlsbad, CA, USA), in accordance with manufacturer's instructions.

\subsubsection{Quality Control of the Sequences and OTU Picking}

Raw sequencing reads were demultiplexed and quality filtered using QIIME 1.9.1 [36]. The reads included presented: a length greater than $225 \mathrm{bp}$; a mean quality score above 25 in a sliding window of 50 nucleotides; no mismatches on the primer; and default values for other quality parameters. The quality-filtered reads were subsequently processed with VSEARCH v1.1 pipeline [37]. An initial dereplication step was applied, then the reads were clustered into operational taxonomic units (OTUs) at $97 \%$ similarity with a de novo approach and, finally, de novo chimera checking was performed with the Uchime function. The raw OTU table was transferred into QIIME 1.9.1 and the taxonomic assignment of representative OTUs was performed using the Ribosomal Database Project (RDP) Classifier [38] against the Greengenes v13.8 database [39]. The sequences were aligned with PyNAST [40]. We sequentially 
applied some extra filtering steps in the aligned and taxonomy-assigned OTU table to filter out sequences belonging to chloroplasts and those belonging to the Shewanellaceae and Halomonadaceae families, which were represented in the NTC and considered as contamination due to the reagents.

\subsubsection{Data Analysis}

Data obtained from relative abundance and reads for each sample were organized at the phylum, class, order, family, and genus levels. The alpha-diversity (within a given sample) was calculated at the genus level using two indexes: the exponential Shannon index [41] (i.e., the number of effective OTUs, or Hill number of order 1) and the inverse Simpson index [42] (i.e., the number of highly effective OTUs, or Hill number of order 2). Two-way analysis of variance (ANOVA) was used to assess the effect of treatment and time on alpha diversity, community stabilization (i.e., beta-diversity compared to the previous day) and acidification activity, with the Bonferroni post-test for pairwise analyses at each time point. The ANOVA was performed and the graphs generated with GraphPad Prism 5 (GraphPad Software Inc, San Diego, CA, USA). Beta-diversity (among samples, either from the same or a different treatment) was studied using two types of multivariate analysis at the genus level: non-metric multidimensional scaling (NMDS) for visual grouping, and analysis of similarities (ANOSIM) for statistical significance. More precisely, the significance of the effect of the treatment and time factors was evaluated with two-way ANOSIM, while pairwise differences between treatments on days 7-14 were evaluated with one-way ANOSIM. This procedure was performed with both the Sorensen [43] and Bray-Curtis [44] beta-diversity indexes, using the PAST 3.0 program [45].

\section{Results}

\subsection{Relative Abundance}

The samples from the three donors (two females and one male aged between 25 and 27) belonged to the Firmicutes-predominant enterotype according to the MetaHit classifier, and a 1:1:1 mixture was therefore prepared as an inoculum for all ex vivo experiments.

As expected, the bacterial communities were dominated by the classes Bacilli, Clostridia, and Erysipelotrichia (all three of which belong to the Firmicutes phylum), Bacteroidia (the Bacteroidetes phylum), Actinobacteria, and Coriobacteriia (the Actinobacteria phylum) and Betaproteobacteria, Deltaproteobacteria, and Gammaproteobacteria (Proteobacteria phylum) (Figure 1); Clostridia and Bacteroidia were the two most abundant classes in the inoculum (Figure 1A). 

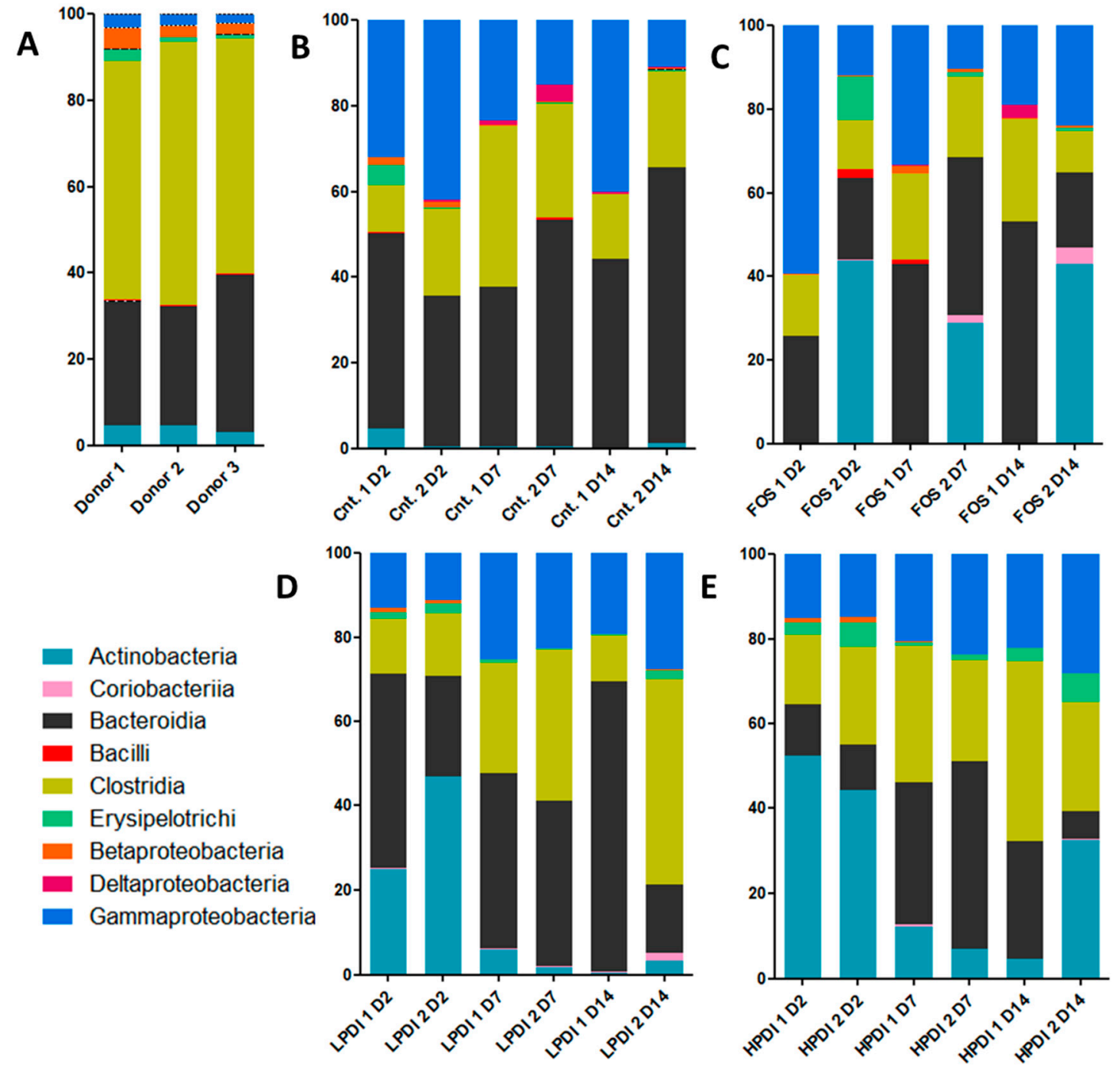

Figure 1. Representation of relative abundance to the class level from the initial time, day 2 (D2), day 7 (D7), and day 14 (D14). Assays were: (A) Sample donors; (B) Controls (Cnt.); (C) Fructooligosaccharides (FOS); (D) Low-polymerization-degree inulin (LPDI); and (E) High-polymerization-degree inulin (HPDI). Each assay was performed in duplicate.

Under all experimental conditions, Gammaproteobacteria were increased, Clostridia were moderately reduced, and Betaproteobacteria were markedly reduced. Coriobacteria, Erysipelotrichia, Bacilli, and Deltaproteobacteria had minor fluctuations over time, except Erysipelotrichia, that were moderately increased with HPDI. Actinobacteria and Bacteroidia continued to be present throughout the fermentation time, but presented considerable fluctuations even among replicates. The control treatment (Macfarlane medium without prebiotic) resulted in the most consistent growth of Bacteroidia, while supplementation with HPDI resulted in the most stable abundance of Clostridia, albeit somewhat reduced compared to the donors' original microbiotas (Figure 1).

\subsection{Alpha-Diversity}

Two indexes to determine diversity were calculated: the exponential Shannon index and the inverse Simpson index, which correspond to Hill numbers 1 and 2, respectively. Broadly similar trends were observed in both indexes (Figure 2). Diversity was markedly reduced on day 2, but tended to stabilization thereafter for most treatments. At the end of the experiment (day 14), HPDI achieved greater recovery of alpha-diversity, given that it almost reached the initial levels as per the inverse 
Simpson index. LPDI and FOS had lower alpha-diversity, and the control treatment (no prebiotic) resulted in the lowest alpha-diversity on day 14. A two-way ANOVA analysis with Bonferroni correction indicated that the only significant pairwise difference was that of HPDI against the control on day $14(p<0.05)$, while all other pairwise comparisons between groups and days were nonsignificant.

A

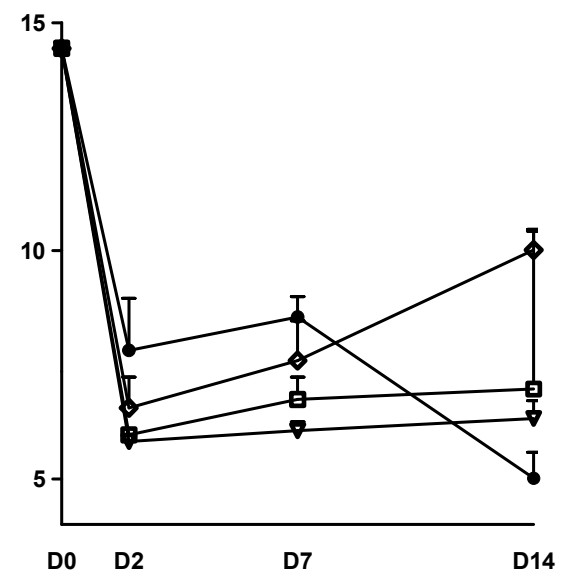

B

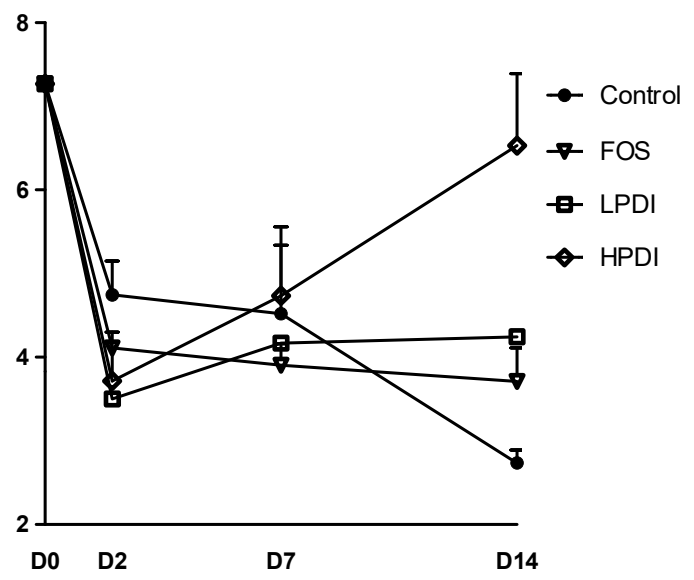

Figure 2. Alpha-diversity at the genus level as per (A) the exponential Shannon index (Hill number 1); and (B) the inverse Simpson index (Hill number 2).

\subsection{Stabilization of the System}

Microbiota changes at the genus level over time and within each treatment were studied with the Sorensen and Bray-Curtis beta-diversity indexes. In general, similarities with respect to the previous time point increased over time, thus indicating a more stable composition (Figure 3). The lowest similarity was observed between days 0 and 2, thereby indicating a marked compositional departure from the inoculum. Greater stability was observed for the Sorensen index than for the Bray-Curtis index, especially on days 2 and 7, thus indicating that the more abundant genera played a more significant role in the differences within each treatment over time, as the Bray-Curtis index gives greater weight to these genera compared to the Sorensen index. The similarity between days 7 and 14 was the highest for all treatments, which would suggest that the microbial community composition stabilized, yet a plateau was not observed within the experimental timeframe. A two-way ANOVA analysis with Bonferroni correction indicated that treatment with FOS resulted in the lowest stability on day 2 (i.e., the largest departure from the inoculum), as per the Sorensen index, compared to all other treatments (Bonferroni $p<0.05$ for the comparison vs control, HPDI and LPDI). Conversely, HPDI had a lower stability than all other treatments on day 14, as per the Bray-Curtis index (Bonferroni $p<0.05$ for the comparisons vs control, FOS and LPDI), thereby suggesting that the more abundant genera had perhaps not yet stabilized. All other pairwise comparisons between groups and days were nonsignificant. 
A

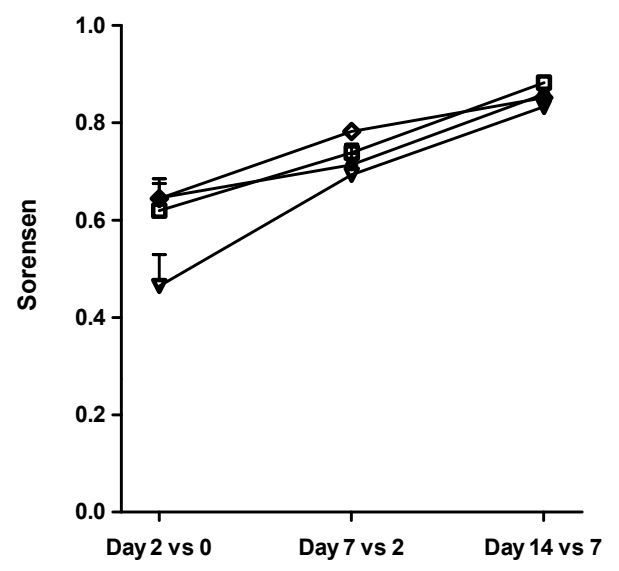

B

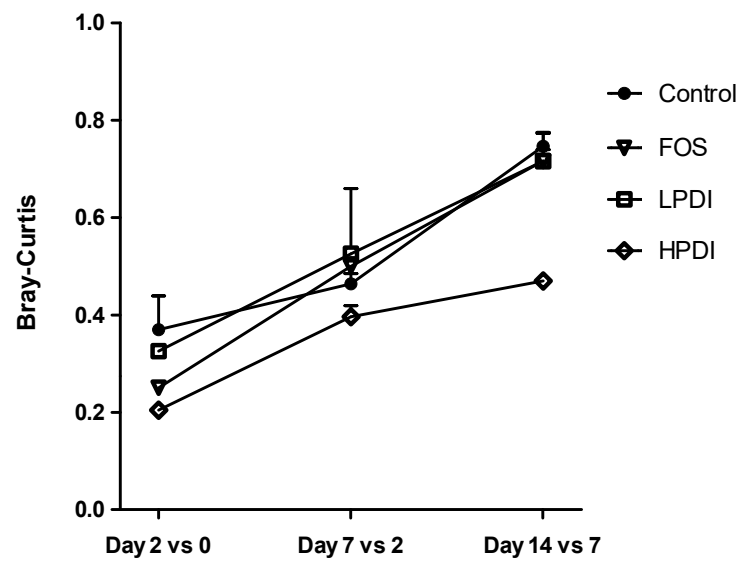

Figure 3. Composition stability, measured as the similarity at the genus level between contiguous sampling days, according to: (A) the Sorensen index; and (B) the Bray-Curtis index.

\subsection{Similarity Between Treatments}

The NMDS analysis revealed a clear closeness between individual donor samples (subsequently pooled as inoculum), but a clear separation between the samples and the microbiotas at days 7 and 14 of ex vivo fermentation, as per both the Sorensen and Bray-Curtis indexes (Figure 4). The Sorensen index clustered together the microbiotas fermented with both the LPDI and HPDI, which were clearly separated from those fermented with FOS or the control medium (no fructan). Conversely, with the Bray-Curtis index, the microbiotas fermented with FOS remained separated, but the LPDI separated further from the HPDI, while becoming closer to the control condition. It is worth noting that sample separation within HPDI in NMDS analysis was markedly larger with the Bray-Curtis index than with the Sorensen one, thus indicating that the variability within HPDI seem to be mostly due to few highly abundant genera.
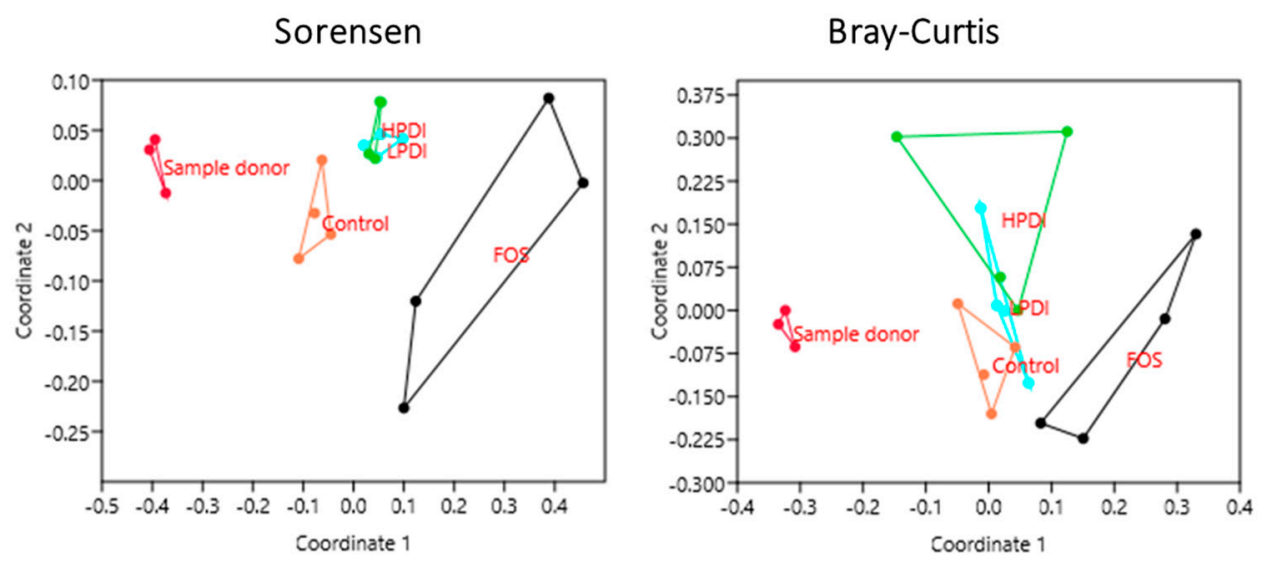

Figure 4. Clustering of samples based on nonmetric multidimensional scaling (NMDS) using the Sorensen and Bray-Curtis indexes at the genus level.

Microbial composition varied significantly both over time and among treatments, as significant differences were observed upon two-way ANOSIM analysis for the time and treatment factors with both the Sorensen ( $p \leq 0.0001$ for both treatment and time) and Bray-Curtis indexes ( $p=0.0045$ and $p \leq 0.0001$ for treatment and time, respectively). A pairwise comparison between treatments in the period between days 7 and 14 indicated that the community structure of microbiotas resulting from fermentation with HPDI or LPDI did not differ significantly from one another, while almost all other pairwise comparisons resulted in nominally significant differences. More differences were noted with 
the Sorensen index than with the Bray-Curtis index, although none of these significances survived the Bonferroni correction (Table 1). Of note, NMDS stress factor was below 0.2 both for Bray-Curtis and Sorensen, indicating that treatment-based clustering was nonrandom.

Table 1. Significance of the differences between treatments based on pooled data from days 7 and 14, one-way ANOSIM using the Sorensen and Bray-Curtis indexes at the genus level. Reported $p$-values are nominal, and none of them held after the Bonferroni correction was applied. vs._-versus.

\begin{tabular}{lcc}
\hline \multicolumn{1}{c}{ Sample } & Sorensen Index $(p$-Value $)$ & Bray-Curtis Index $(p$-Value $)$ \\
\hline Sample Donor vs. Control & 0.0261 & 0.0296 \\
Sample Donor vs. FOS & 0.0286 & 0.0285 \\
Sample Donor vs. LPDI & 0.0259 & 0.0301 \\
Sample Donor vs. HPDI & 0.0303 & 0.0290 \\
Control vs. FOS & 0.0274 & 0.0282 \\
Control vs. LPDI & 0.0298 & 0.1477 \\
Control vs. HPDI & 0.0283 & 0.0284 \\
FOS vs. LPDI & 0.0308 & 0.0283 \\
FOS vs. HPDI & 0.0282 & 0.0818 \\
LPDI vs. HPDI & 0.2602 & 0.1952 \\
\hline
\end{tabular}

\section{5. $p H$ Variation}

Analysis of the differential amount of $\mathrm{NaOH}$ and $\mathrm{HCl}$ consumed to maintain the fermenter in the correct $\mathrm{pH}$ range (Figure 5) showed that all treatments induced acidification over time $(p<0.0001)$, with significant differences among the treatments $(p=0.0012)$. LPDI and HPDI did not differ from one another, but clearly produced more acidification than either the control or FOS on days 7 and 14 (Bonferroni post-test $p$-value $<0.01$ for all pairwise comparisons). Note that acidification with FOS was in fact found to be lower than the control on day 14 (Bonferroni $p<0.05$ ).

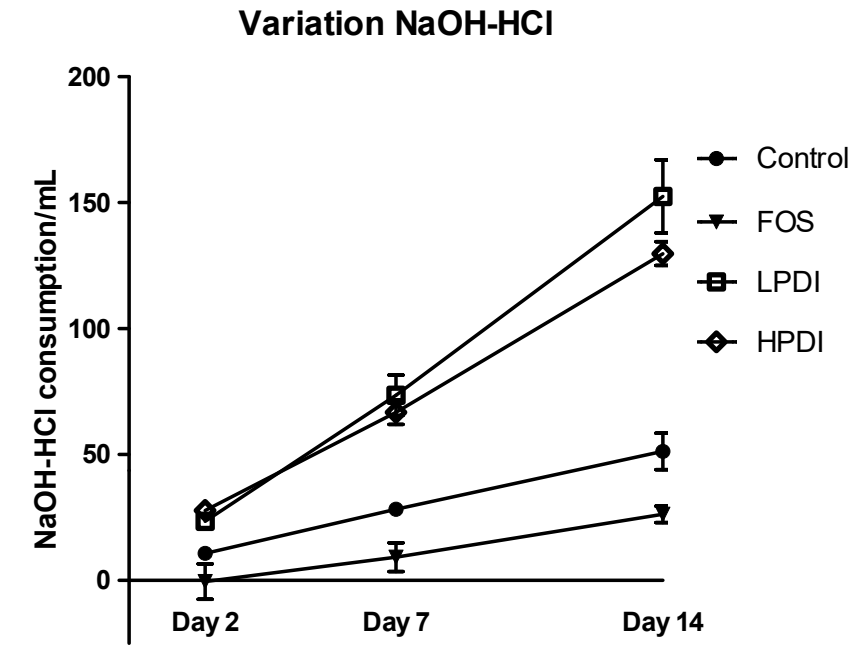

Figure 5. Total acidification activity, expressed as the difference in $\mathrm{mL}$ of the consumption of $\mathrm{NaOH}$ and $\mathrm{HCl}$ required to keep the system's $\mathrm{pH}$ stable, for the different fructans (FOS, LPDI, and HPDI) and the control treatment.

\section{Discussion}

The health benefits of prebiotics are widely accepted [46], and the present study provides further evidence that fructan-type prebiotics can modify the human intestinal microbiota. However, fructans with differing degrees of polymerization (FOS, LPDI, and HPDI) had different impacts on the evolution of the microbial community. 
Our study used a simple system to simulate average colonic conditions and test the effects of fructans with different degrees of polymerization in the human gut microbiota. More precisely, our system corresponded to the transverse colon (middle stage), based on a system developed by Macfarlane, with $\mathrm{pH}$ and retention time conditions similar to the transverse colon conditions in the SHIME [21]. Conversely, Lacroix [47], with three stages, used a shorter retention time because it simulated the digestive tract of children. The fermenters were inoculated with pooled fecal material from three donors belonging to the same Firmicutes enterotype, which has been reported to be the most common in the population [26]. Moreover, the relative abundances of the microbial communities in the inocula were similar to those described in the literature for healthy patients $[8,26]$.

This study showed that all alpha-diversity indexes initially decreased in all treatments. Recent studies that compared the effect of three commercial apple varieties (grated with the skin) versus inulin and cellulose [48], and the effects of FOS, GOS, XOS, and beta-glucan [49], also showed decreased alpha-diversity over time for all treatments. This could be a common effect of ex vivo simulation compared to the real human gut. The results of So et al. 2018 [17] also support the selectivity criteria of the prebiotic concept, in which the host microorganisms selectively utilize the prebiotic as substrates. It could be hypothesized that the loss in diversity is due to fructans stimulating just a few bacterial groups, but our results showed that the control without prebiotic brought about the same behavior, which would rule out fructans as the cause of diversity loss. The metabolization of dietary polysaccharides by the gastrointestinal bacteria is an example of the symbiotic relationship between the host and the microbiota. Furthermore, this relationship provides an avenue for dietary modulation of the microbiota, because microbial growth and metabolism depend on substrate availability, e.g., the type of dietary fiber or prebiotic consumed by the host [20]. The Simpson index (i.e., number of highly effective OTUs) gives more weight to abundant OTUs, compared to the inverse Shannon index (i.e., the number of effective OTUs). Our system showed that diversity decreased with both indexes and, consequently, in both abundant and rare OTUs. Differences among these two indexes showed that fructans with a higher degree of polymerization (HDPI) produced more diversity towards the end of the experiment, and that this effect was statistically significant against controls when more weight was attached to the more abundant (or "highly effective") OTUs.

Results obtained with our ex vivo fermentation system suggest that the microbiota stabilized from day 7 of fermentation, as the similarity between days 7 and 14 was found to be high, although a plateau was not yet reached or not detected. Macfarlane reported that his system stabilized in $24 \mathrm{~h}$, but only nine bacterial groups where checked with PCR [25]. Conversely, the SHIME takes up to two weeks to stabilize when 130 different bacterial groups are considered [23], a result much more consistent with our findings. Greater stability was observed with the Sorensen index compared to Bray-Curtis index, thus indicating that low-abundance OTUs stabilized faster than highly abundant OTUs, akin to the behavior of alpha-diversity indexes. Moreover, stability after 14 days of highly abundant OTUs was significantly lower for long-chain fructans (HPDI) than for all other treatments. Together with the higher alpha-diversity attained compared to controls, we hypothesize that, given more time, HDPI could produce an even greater increase in alpha diversity.

As expected, differences in the specific microbiota composition were found among treatment groups. However, Bonferroni-corrected statistical significance was not attained in pairwise comparisons, probably owing to the large number of between-group comparisons (six) and the limited number of replicas.

Taken together, our results show that fructans with a polymerization degree of above 10 (HPDI and LPDI) result in similar microbiota compositions, especially with respect to low-abundant OTUs, and in similarly high acidification activity, compared to the control condition (no fructans). Moreover, the fructan with the highest degree of polymerization (HPDI) restored alpha-diversity to baseline levels compared to the control when more weight was given to highly abundant OTUs (inverse Simpson index). These highly abundant OTUs were Bifidobacterium, Bacteroides, Prevotella, Faecalibacterium, Roseburia, Acidaminococcus, Megasphaera, Catenibacterium, Citrobacter, and three undefined genera 
belonging to the families Enterobacteriaceae, Lachnospiraceae, and Eubacteriaceae. Our observations also highlight the importance of using different diversity indexes to reveal the differences between more abundant and less abundant OTUs under a given study factor. Moreover, our experiment showed that FOS (polymerization degree 2 to 8 ) promoted a different microbial composition than longer-chain inulins, and failed to stimulate acidification activity compared to control fermentation without fructans.

Numerous reports link a decreased diversity of the gut microbiota to a declined health status, including conditions such as inflammatory bowel diseases (ulcerative colitis and Crohn's disease), coeliac disease, allergies, and obesity [50]. Recent simulation studies support a causative role of microbiota diversity in health status [51], and drops in diversity have been associated to a higher risk of subsequent recurrence both in ulcerative colitis [52,53] and Crohn's disease [54,55]. Previous studies in inflammatory bowel disease have used mixtures of low and medium length inulin-type fructans (i.e., FOS and LDPI) [56]. However, based on our results, HDPI seems a better candidate to promote microbiota diversity to help prevent recurrence in inflammatory bowel disease, and deserves further testing in clinical trials.

One of the limitations of our ex vivo model was our use of a single stage of colonic conditions, corresponding to the transverse colon, rather than multiple stages to simulate the functionality of different locations in the gut. As fibers are normally degraded in the gut, this factor could introduce additional differences not observed in this study. A second limitation of our study was the small number of replicates performed for each treatment, which reduced the statistical power in some analyses. A third limitation was the fact that the effects of the polymerization degree of fructans were tested on a microbiota with the Firmicutes-predominant enterotype, and future studies on other enterotypes are required to compare the results. Any future research should also measure the production of individual short-chain fatty acids in order to gain further information on the effect of the polymerization degree on the metabolic activity of the microbiota, which in our study was limited to total acidification activity. Such research could reveal additional similarities or differences between different fructans. Finally, it would be especially interesting to study the effect of the degree of polymerization when different fructans are combined, or even when other types of fiber such as beta-glucans, polydextrose, acacia gum, pectin, and partially hydrolyzed guar gum are added. The combination of different fibers could be important to avoid feeding a few bacterial groups, and thus promote a more diverse microbiota [20].

\section{Conclusions}

In conclusion, the ex vivo system used in our study allowed us to observe the effect of the degree of polymerization of fructans on the intestinal microbiota. The degree of polymerization of fructans seems to impact their prebiotic effect, resulting in microbiotas with different compositions. Higher polymerization seems to facilitate greater diversity and acidification activity compared to lower polymerization (i.e., FOS), suggesting that inulins of different degree of polymerization produce different effects on the gut microbiota, both at the compositional and metabolic level. Ultimately, adequately powered clinical trials should be conducted to confirm these effects in humans.

Supplementary Materials: The following are available online at http:/www.mdpi.com/2072-6643/11/6/1293/s1, Table S1: Dietary Questionnaire.

Author Contributions: Conceptualization: E.A., I.M. and J.C.; investigation: E.A., I.M., M.R.-P. and J.C.; research design: E.A., I.M. and J.E.; data curation: E.A. and J.E.; analysis and writing of the manuscript: E.A., A.F.-C. and J.E. All authors approved the final version of the manuscript.

Funding: This research was funded by the Secretary for Universities and Research under the Ministry of Economy and Knowledge of the Generalitat de Catalunya (award no.: 2014 DI 073).

Conflicts of Interest: The authors declare no conflicts of interest regarding the publication of this paper. 


\section{References}

1. Salminen, S.; Bouley, C.; Boutron, M.-C.; Cummings, J.H.; Franck, A.; Gibson, G.R.; Isolauri, E.; Moreau, M.-C.; Roberfroid, M.; Rowland, I. Functional food science and gastrointestinal physiology and function. Br. J. Nutr. 1998, 80, S147-S171. [CrossRef]

2. Ley, R.E.; Peterson, D.A.; Gordon, J.I. Ecological and evolutionary forces shaping microbial diversity in the human intestine. Cell 2006, 124, 837-848. [CrossRef] [PubMed]

3. Gilbert, J.A.; Blaser, M.J.; Caporaso, J.G.; Jansson, J.K.; Lynch, S.V.; Knight, R. Current understanding of the human microbiome. Nat. Med. 2018, 24, 392-400. [CrossRef] [PubMed]

4. Backhed, F. Host-Bacterial Mutualism in the Human Intestine. Science 2005, 307, 1915-1920. [CrossRef] [PubMed]

5. Huttenhower, C.; Gevers, D.; Knight, R.; Abubucker, S.; Badger, J.H.; Chinwalla, A.T.; Creasy, H.H.; Earl, A.M.; Fitzgerald, M.G.; Fulton, R.S.; et al. Structure, function and diversity of the healthy human microbiome. Nature 2012, 486, 207-214. [CrossRef]

6. Qin, J.; Li, R.; Raes, J.; Arumugam, M.; Burgdorf, S.; Manichanh, C.; Nielsen, T.; Pons, N.; Yamada, T.; Mende, D.R.; et al. A human gut microbial gene catalog established by metagenomic sequencing. Nature 2010, 464, 59-65. [CrossRef]

7. Zeevi, D.; Korem, T.; Godneva, A.; Bar, N.; Kurilshikov, A.; Lotan-Pompan, M.; Weinberger, A.; Fu, J.; Wijmenga, C.; Zhernakova, A.; et al. Structural variation in the gut microbiome associates with host health. Nature 2019, 568, 43-48. [CrossRef]

8. $\quad$ Eckburg, P.B.; Bik, E.M.; Berstein, C.N.; Purdom, E.; Dethlefsen, L.; Sargent, M.; Gill, S.R.; Nelson, K.E.; Relman, D.A. Diversity of the human intestinal microbial flora. Science 2005, 308, 1635-1638. [CrossRef]

9. Rowland, I.; Gibson, G.; Heinken, A.; Scott, K.; Swann, J.; Thiele, I.; Tuohy, K. Gut microbiota functions: Metabolism of nutrients and other food components. Eur. J. Nutr. 2018, 57, 1-24. [CrossRef]

10. Flint, H.J.; Scott, K.P.; Louis, P.; Duncan, S.H. The role of the gut microbiota in nutrition and health. Nat. Rev. Gastroenterol. Hepatol. 2012, 9, 577-589. [CrossRef]

11. Gibson, G.R.; Roberfroid, M.B. Dietary modulation of the human colonic microbiota: Introducing the concept of prebiotics. J. Nutr. 1995, 125, 1401-1412. [CrossRef] [PubMed]

12. Gibson, G.R.; Hutkins, R.; Sanders, M.E.; Prescott, S.L.; Reimer, R.A.; Salminen, S.J.; Scott, K.; Stanton, C.; Swanson, K.S.; Cani, P.D.; et al. Expert consensus document: The International Scientific Association for Probiotics and Prebiotics (ISAPP) consensus statement on the definition and scope of prebiotics. Nat. Rev. Gastroenterol. Hepatol. 2017, 14, 491-502. [CrossRef] [PubMed]

13. De Vrese, M.; Schrezenmeir, J. Probiotics, Prebiotics, and Synbiotics. Adv. Biochem. Eng. Biotechnol. 2008, 111, 1-66. [CrossRef] [PubMed]

14. Kelly, G. Inulin-Type Prebiotics-A Review: Part 1. Altern. Med. Rev. 2008, 13, 315-329. [PubMed]

15. Gibson, G.R.; Beatty, E.R.; Wang, X.I.N.; Cummings, J.H. Selective Stimulation of Bifidobacteria in the Human Colon by Oligofructose and Inulin. Gastroenterology 1995, 108, 975-982. [CrossRef]

16. Gibson, G.R.; Wang, X. Bifidogenic properties of different types of fructo-oligosaccharides. Food Microbiol. 1994, 11, 491-498. [CrossRef]

17. So, D.; Whelan, K.; Rossi, M.; Morrison, M.; Holtmann, G.; Kelly, J.T.; Shanahan, E.R.; Staudacher, H.M.; Campbell, K.L. Dietary fiber intervention on gut microbiota composition in healthy adults: A systematic review and meta-analysis. Am. J. Clin. Nutr. 2018, 107, 965-983. [CrossRef] [PubMed]

18. Scott, K.P.; Martin, J.C.; Duncan, S.H.; Flint, H.J. Prebiotic stimulation of human colonic butyrate-producing bacteria and bifidobacteria, in vitro. FEMS Microbiol. Ecol. 2014, 87, 30-40. [CrossRef]

19. Poeker, S.A.; Geirnaert, A.; Berchtold, L.; Greppi, A.; Krych, L.; Steinert, R.E.; De Wouters, T.; Lacroix, C. Understanding the prebiotic potential of different dietary fibers using an in vitro continuous adult fermentation model (PolyFermS). Sci. Rep. 2018, 8, 4318. [CrossRef]

20. Holscher, H.D. Dietary fiber and prebiotics and the gastrointestinal microbiota. Gut Microbes 2017, 8, 172-184. [CrossRef]

21. Venema, K.; Van Den Abbeele, P. Experimental models of the gut microbiome. Best Pract. Res. Clin. Gastroenterol. 2013, 27, 115-126. [CrossRef] [PubMed] 
22. Molly, K.; Woestyne, M.V.; Smet, I.D.; Verstraete, W. Validation of the Simulator of the Human Intestinal Microbial Ecosystem (SHIME) Reactor Using Microorganism-associated Activities. Microb. Ecol. Health Dis. 1994, 7, 191-200. [CrossRef]

23. Van Den Abbeele, P.; Grootaert, C.; Marzorati, M.; Possemiers, S.; Verstraete, W.; Gérard, P.; Rabot, S.; Bruneau, A.; Aidy Ei, S.; Derrien, M.; et al. Microbial community development in a dynamic gut model is reproducible, colon region specific, and selective for bacteroidetes and Clostridium cluster IX. Appl. Environ. Microbiol. 2010, 76, 5237-5246. [CrossRef] [PubMed]

24. Minekus, M.; Smeets-Peeters, M.; Bernalier, A.; Marol-Bonnin, S.; Havenaar, R.; Marteau, P.; Alric, M.; Fonty, G. A computer-controlled system to simulate conditions of the large intestine with peristaltic mixing, water absorption and absorption of fermentation products. Appl. Microbiol. Biotechnol. 1999, 53, 108-114. [CrossRef] [PubMed]

25. Macfarlane, G.T.; Macfarlane, S.; Gibson, G.R. Validation of a three-stage compound continuous culture system for investigating the effect of retention time on the ecology and metabolism of bacteria in the human colon. Microb. Ecol. 1998, 35, 180-187. [CrossRef] [PubMed]

26. Costea, P.I.; Hildebrand, F.; Manimozhiyan, A.; Bäckhed, F.; Blaser, M.J.; Bushman, F.D.; de Vos, W.M.; Ehrlich, S.D.; Fraser, C.M.; Hattori, M.; et al. Enterotypes in the landscape of gut microbial community composition. Nat. Microbiol. 2018, 3, 8-16. [CrossRef] [PubMed]

27. Arumugam, M.; Raes, J.; Pelletier, E.; Le Paslier, D.; Yamada, T.; Mende, D.R.; Fernandes, G.R.; Tap, J.; Bruls, T.; Batto, J.M.; et al. Enterotypes of the human gut microbiome. Nature 2011, 473, 174-180. [CrossRef] [PubMed]

28. Ding, T.; Schloss, P.D. Dynamics \& Associations of Microbial Organism Types Across the Human Body. Nature 2014, 509, 357-360. [CrossRef]

29. Wu, G.D.; Chen, J.; Hoffmann, C.; Bittinger, K.; Chen, Y.; Keilbaugh, S.A.; Bewtra, M.; Knights, D.; Walters, W.A.; Knight, R.; et al. Linking Long-Term Dietary Patterns with Gut Microbial Enterotypes. Science 2011, 334, 105-109. [CrossRef]

30. Macfarlane, G.T.; Macfarlane, S. Models for intestinal fermentation: Association between food components, delivery systems, bioavailability and functional interactions in the gut. Curr. Opin. Biotechnol. 2007, 18, 156-162. [CrossRef]

31. Riegler, G.; Espopito, I. Bristol scale stool form. A still valid help in medical practice and clinical research. Tech. Coloprotocol. 2001, 5, 163-164. [CrossRef] [PubMed]

32. Aguirre, M.; Ramiro-Garcia, J.; Koenen, M.E.; Venema, K. To pool or not to pool? Impact of the use of individual and pooled fecal samples for in vitro fermentation studies. J. Microbiol. Methods 2014, 107, 1-7. [CrossRef] [PubMed]

33. Aguirre, M.; Eck, A.; Koenen, M.E.; Savelkoul, P.H.M.; Budding, A.E.; Venema, K. Evaluation of an optimal preparation of human standardized fecal inocula for in vitro fermentation studies. J. Microbiol. Methods 2015, 117, 78-84. [CrossRef] [PubMed]

34. Gibson, G.R.; Cummings, J.H.; Macfarlane, G.T. Use of a three-stage continuous culture system to study the effect of mucin on dissimilatory sulfate reduction and methanogenesis by Use of a Three-Stage Continuous Culture System To Study the Effect of Mucin on Dissimilatory Sulfate Reduction and Methano. Appl. Environ. Microbiol. 1988, 54, 2750-2755. [PubMed]

35. Steed, H.; MacFarlane, G.T.; Blackett, K.L.; Bahrami, B.; Reynolds, N.; Walsh, S.V.; Cummings, J.H.; MacFarlane, S. Clinical trial: The microbiological and immunological effects of synbiotic consumption - A randomized double-blind placebo-controlled study in active Crohn's disease. Aliment. Pharmacol. Ther. 2010, 32, 872-883. [CrossRef] [PubMed]

36. Caporaso, J.G.; Kuczynski, J.; Stombaugh, J.; Bittinger, K.; Bushman, F.D.; Costello, E.K.; Fierer, N.; Peña, A.G.; Goodrich, J.K.; Gordon, J.I.; et al. QIIME allows analysis of high-throughput community sequencing data. Nat. Methods 2010, 7, 335-336. [CrossRef] [PubMed]

37. Rognes, T.; Flouri, T.; Nichols, B.; Quince, C.; Mahé, F. VSEARCH: A versatile open source tool for metagenomics. PeerJ 2016, 4, e2584. [CrossRef]

38. Wang, Q.; Garrity, G.M.; Tiedje, J.M.; Cole, J.R. Navie Bayesian classifier for rapid assignment of rRNA sequences into the new bacterial taxonomy. Appl. Environ. Microbiol. 2007, 73, 5261-5267. [CrossRef] [PubMed] 
39. DeSantis, T.Z.; Hugenholtz, P.; Larsen, N.; Rojas, M.; Brodie, E.L.; Keller, K.; Huber, T.; Dalevi, D.; Hu, P.; Andersen, G.L. Greengenes, a chimera-checked $16 \mathrm{~S}$ rRNA gene database and workbench compatible with ARB. Appl. Environ. Microbiol. 2006, 72, 5069-5072. [CrossRef]

40. Caporaso, J.G.; Bittinger, K.; Bushman, F.D.; Desantis, T.Z.; Andersen, G.L.; Knight, R. PyNAST: A flexible tool for aligning sequences to a template alignment. Bioinformatics 2010, 26, 266-267. [CrossRef]

41. Shannon, C.E. A Mathematical Theory of Communication. Bell Syst. Tech. J. 1948, 27, 379-423. [CrossRef]

42. Simpson, E.H. Measurement of Diversity. Nature 1949, 163, 688. [CrossRef]

43. Sorensen, T. A Method of Establishing Groups of Equal Amplitude in Plant Sociology Based on Similarity of Species and Its Application to Analyses of the Vegetation on Danish Commons. Biol. Skr. 1948, 5, 1-34.

44. Bray, J.R.; Curtis, J.T. An Ordination of the Upland Forest Communities of Southern Wisconsin. Ecol. Monogr. 1957, 27, 325-349. [CrossRef]

45. Hammer, Ø.; Harper, D.; Ryan, P. Past: Paleontological statistics software package for education and data analysis. Paleontol. Electron. 2001, 4, 9. [CrossRef]

46. Carlson, J.L.; Erickson, J.M.; Hess, J.M.; Gould, T.J.; Slavin, J.L. Prebiotic dietary fiber and gut health: Comparing the in vitro fermentations of beta-glucan, inulin and xylooligosaccharide. Nutrients 2017, 9, 1361. [CrossRef] [PubMed]

47. Cinquin, C.; Le Blay, G.; Fliss, I.; Lacroix, C. New three-stage in vitro model for infant colonic fermentation with immobilized fecal microbiota. FEMS Microbiol. Ecol. 2006, 57, 324-336. [CrossRef] [PubMed]

48. Koutsos, A.; Lima, M.; Conterno, L.; Gasperotti, M.; iBanchi, M.; Fava, F.; Vrhovsek, U.; Lovegrove, J.A.; Tuohy, K.M. Effects of commercial apple varieties on human gut microbiota composition and metabolic output using an in vitro colonic model. Nutrients 2017, 9, 533. [CrossRef]

49. Fehlbaum, S.; Prudence, K.; Kieboom, J.; Heerikhuisen, M.; van den Broek, T.; Schuren, F.H.J.; Steinert, R.E.; Raederstorff, D. In vitro fermentation of selected prebiotics and their effects on the composition and activity of the adult gut microbiota. Int. J. Mol. Sci. 2018, 19, 3097. [CrossRef]

50. Mosca, A.; Leclerc, M.; Hugot, J.P. Gut microbiota diversity and human diseases: Should we reintroduce key predators in our ecosystem? Front. Microbiol. 2016, 7, 1-12. [CrossRef]

51. Larsen, O.F.A.; Claassen, E. The mechanistic link between health and gut microbiota diversity. Sci. Rep. 2018, 8, 6-10. [CrossRef] [PubMed]

52. Ott, S.J.; Plamondon, S.; Hart, A.; Begun, A.; Rehman, A.; Kamm, M.A.; Schreiber, S. Dynamics of the mucosa-associated flora in ulcerative colitis patients during remission and clinical relapse. J. Clin. Microbiol. 2008, 46, 3510-3513. [CrossRef] [PubMed]

53. Shen, Z.-H.; Zhu, C.-X.; Quan, Y.-S.; Yang, Z.-Y.; Wu, S.; Luo, W.-W.; Tan, B.; Wang, X.-Y. Relationship between intestinal microbiota and ulcerative colitis: Mechanisms and clinical application of probiotics and fecal microbiota transplantation. World J. Gastroenterol. 2018, 24, 5-14. [CrossRef] [PubMed]

54. Dey, N.; Soergel, D.A.W.; Repo, S.; Brenner, S.E. Association of gut microbiota with post-operative clinical course in Crohn's disease. BMC Gastroenterol. 2013, 13, 131. [CrossRef] [PubMed]

55. Pascal, V.; Pozuelo, M.; Borruel, N.; Casellas, F.; Campos, D.; Santiago, A.; Martinez, X.; Varela, E.; Sarrabayrouse, G.; Machiels, K.; et al. A microbial signature for Crohn's disease. Gut 2017, 66, 813-822. [CrossRef] [PubMed]

56. Rasmussen, H.E.; Hamaker, B.R. Prebiotics and Inflammatory Bowel Disease. Gastroenterol. Clin. 2017, 46, 783-795. [CrossRef]

(C) 2019 by the authors. Licensee MDPI, Basel, Switzerland. This article is an open access article distributed under the terms and conditions of the Creative Commons Attribution (CC BY) license (http://creativecommons.org/licenses/by/4.0/). 